\title{
Real-time measurement system for determining metal concentrations in water-intensive processes
}

https://doi.org/10.1515/eng-2020-0063

Received Oct 10, 2019; accepted Apr 08, 2020

\begin{abstract}
The main purpose of this paper is to demonstrate the potential of time-domain nuclear magnetic resonance (TD-NMR) technology for monitoring the concentrations of metal ions in water-based solutions. The main focus of this work was paramagnetic ions, such as $\mathrm{Mn}^{2+}, \mathrm{Cu}^{2+}$, $\mathrm{Fe}^{3+}, \mathrm{Fe}^{2+}, \mathrm{Zn}^{2+}$ and $\mathrm{Ni}^{2+}$, which are often the principal metal components in mining waters. Laboratory samples of different concentrations of single metals and mixtures of them and samples of real mining water were used in the relaxation rate $\left(R_{2}\right)$ measurements. The measurements of single metal ions were used for the determination of the relaxivities of those ions. The concentrations of the ions in the mining water as a function of $\mathrm{pH}$ were also estimated by means of the X-ray fluorescence (XRF) method and ChemEQL software for calculating chemical speciation equilibria. Using these concentration values and the relaxivities of the metal ions, the total relaxation rate $\left(R_{2}\right)$ results were then calculated. Principally, the results of these three different determinations are in relatively good agreement. It can be concluded that TD-NMR has great potential for monitoring metal ion concentrations during water treatment.
\end{abstract}

Keywords: NMR, time-domain, metal concentration, precipitation, mining water

\section{Introduction}

Tightening requirements for the environmental quality of mining and process waters require more efficient purifi-

\footnotetext{
`Corresponding Author: Yrjö Hiltunen: FiberLaboratory, South Eastern Finland University of Applied Sciences, Savonlinna, 57200, Finland; Email: yrjo.hiltunen@xamk.fi

Ekaterina Nikolskaya: FiberLaboratory, South-Eastern Finland University of Applied Sciences, Savonlinna, 57200, Finland; Email: ekaterina.nikolskaya@xamk.fi

Mika Liukkonen: University of Eastern Finland, Kuopio, 70210, Finland; Email: mika.liukkonen@uef.fi
}

cation methods. However, today's commercially available water quality measurements are not able to cope with certain significant contaminants such as metal and sulfate in real time. In addition, the presently used water quality monitoring systems require regular maintenance and calibration, which reduces their cost efficiency.

Chemical precipitation is a conventional technology used to treat mining waters [1]. Chemical precipitation processes involve the addition of chemical reagents, followed by the separation of precipitated solids from clean water. Typically, separation occurs in a clarifier, although separation by filtration or membranes is also possible. Chemical precipitation can also be applied in water pools, in which case the precipitated solids can simply be left at the bottom of the pool. Precipitation can be induced by the addition of an alkali, sulfide, coagulant, or other reagent that will bond with dissolved metal ions. Raising the $\mathrm{pH}$ with the use of alkaline reagents, such as sodium hydroxide, causes certain dissolved metals to precipitate as hydroxides.

Control of the effectiveness of heavy metal removal is essential in different applications [2-4]. It is important and necessary to use fast and accurate analytical methods that can be performed in real time. X-ray fluorescence (XRF) and atomic absorption spectrometry are typically used for heavy metal estimation. The nuclear magnetic resonance (NMR) technique could be an alternative to these methods. It was demonstrated that the limit of quantification of time-domain nuclear magnetic resonance (TD-NMR) is one order of magnitude larger than that of atomic absorption spectrometry; however, TD-NMR is a more robust and less expensive technique [5]. The main advantages of NMR are that it does not destroy the sample, it can be automated, and it is a non-fouling method.

High-resolution NMR has been extensively used in analytical chemistry, but in practice, it is difficult to apply to online process control due to its open magnetic field, large area of influence, high price and required cryogen use. On the other hand, the simpler TD-NMR method is becoming attractive for industrial applications due to its relatively low price, mobility, ease of operation, and simple sample preparation procedure [6-8].

¿ Open Access. (C) 2020 E. Nikolskaya et al., published by De Gruyter. (cc) BY 4.0 License 
TD-NMR is broadly applicable in practical use, and it could potentially be utilized for intensive water process characterization, as NMR relaxation rates are sensitive to paramagnetic ions present in solutions. For example, NMR relaxation times were used to determine the concentrations of various paramagnetic ions $\left(\mathrm{Co}^{2+}, \mathrm{Cr}^{3+}, \mathrm{Cu}^{2+}, \mathrm{Fe}^{3+}\right.$ and $\left.\mathrm{Mn}^{2+}\right)[9,10]$ and the solubility product of paramagnetic cations $\left(\mathrm{Fe}^{3+}, \mathrm{Cu}^{2+}\right.$ and $\left.\mathrm{Mn}^{2+}\right)$ [11] and to investigate the precipitation of mixtures of paramagnetic ions as a function of $\mathrm{pH}[10,11]$. Several investigations were performed by in situ TD-NMR [9, 12] by placing the sample on the NMR probe, and the NMR parameters were measured continuously during the reaction or process.

Despite the mentioned advantages and broad applicability, TD-NMR is still rarely applied online [13]. Monitoring of mining water during its processing was tested by online TD-NMR for the first time in a real mine [14]. TD-NMR was also recently applied to the online analysis of several liquid materials during different processes, such as fatty acid mixing $[15,16]$, starch gelatinization [17] and black liquor evaporation [18]. Moreover, there is a high demand on process analytical tools in various industrial areas nowadays, and novel sensors, developed on the basis of NMR technology, could be beneficial [19-21].

In this paper, TD-NMR technology has been utilized for monitoring the concentrations of paramagnetic metal ions, such as $\mathrm{Mn}^{2+}, \mathrm{Cu}^{2+}, \mathrm{Fe}^{2+}, \mathrm{Fe}^{3+}$ and $\mathrm{Zn}^{2+}$, in waterbased solutions. Different concentrations of single metals and mixtures of them and real mining water samples were used in the tests. First, the relaxivities of separate metal ions were determined by NMR. Then, the concentrations of the single metals were estimated by NMR during the precipitation reactions. Furthermore, the approach was demonstrated in the laboratory during the precipitation reactions of multiple ions in a simple mixture of metals and in real mining water. Additionally, simulation of the metal concentrations during this process was performed using ChemEQL software for calculating chemical speciation equilibria. Monitoring of the $\mathrm{Mn}^{2+}$ concentration in the output mining water was also demonstrated.

\section{Materials and methods}

\subsection{Metal ion samples}

Measurements were performed using samples made in a laboratory and samples of real mining water. The laboratory samples for the relaxivity measurements of single metal ions were made by adding compounds, including appropriate metal ions to drinking water. First, the $\mathrm{pH}$ of the water samples was adjusted to $\mathrm{pH} 1$. Then, the concentrations of metal ions were varied so that their relaxivities could be estimated by a linear function. The measurements were performed for the following ions: $\mathrm{Mn}^{2+}$, $\mathrm{Fe}^{3+}, \mathrm{Fe}^{2+}, \mathrm{Cu}^{2+}$ and $\mathrm{Ni}^{2+}$. In addition, the relaxivities of $\mathrm{Cu}^{2+}$ were determined using two different $\mathrm{Cu}$ compounds, namely, $\mathrm{CuSO}_{4}$ and $\mathrm{CuCl}_{2}$.

For the mining water, the concentrations of metal ions were studied as a function of $\mathrm{pH}$, which was increased by adding sodium hydroxide $(\mathrm{NaOH})$. In the experiment, the $\mathrm{pH}$ level was increased from $\mathrm{pH} 4.6$ to $\mathrm{pH} 12$. The metal concentrations of the samples (in $\mathrm{mg} / \mathrm{l}$ ) were measured by the XRF method. Because the XRF method has certain problems detecting light atoms (e.g., $\mathrm{Mg}$ and $\mathrm{Al}$ ), the concentrations were determined for $\mathrm{Mn}, \mathrm{Fe}, \mathrm{Cu}$ and $\mathrm{Zn}$. In addition, the effects of $\mathrm{Mg}$ and $\mathrm{Al}$ ions on our NMR measurements are very small compared to those of other metal ions, and their concentrations are constant as a function of $\mathrm{pH}$, which also supports the decision to leave these ions out of the analysis. The $\mathrm{pH}$ values and the metal concentrations of the samples are given in Table 1 and Figure 1.

Table 1: Concentrations of metals ( $\mathrm{Mn}, \mathrm{Fe}, \mathrm{Cu}$ and $\mathrm{Zn}$ ) in mine water samples (in $\mathrm{mg} / \mathrm{l}$ ) measured by XRF.

\begin{tabular}{ccccc}
\hline pH & $\begin{array}{c}\text { Mn } \\
\mathbf{m g} / \mathbf{l}\end{array}$ & $\begin{array}{c}\mathrm{Fe} \\
\mathrm{mg} / \mathrm{l}\end{array}$ & $\begin{array}{c}\mathrm{Cu} \\
\mathrm{mg} / \mathbf{l}\end{array}$ & $\begin{array}{c}\mathrm{Zn} \\
\mathrm{mg} / \mathrm{l}\end{array}$ \\
\hline 4.62 & 11.98 & 6.206 & 2.325 & 15.051 \\
5.61 & 11.689 & 4.399 & 1.873 & 15.194 \\
6.6 & 11.705 & 0.853 & 0.081 & 9.812 \\
7.62 & 8.971 & 0 & 0.008 & 0.294 \\
8.15 & 7.678 & 0 & 0.005 & 0.045 \\
8.73 & 6.49 & 0 & 0.006 & 0.018 \\
8.97 & 5.349 & 0.015 & 0.014 & 0.013 \\
9.12 & 4.021 & 0 & 0.009 & 0.013 \\
9.22 & 2.1 & 0 & 0.007 & 0.005 \\
9.38 & 0.82 & 0 & 0.005 & 0.203 \\
9.57 & 0.149 & 0.008 & 0.007 & 0.007 \\
9.82 & 0.036 & 0.013 & 0.01 & 0.017 \\
10 & 0.011 & 0.018 & 0.006 & 0.007 \\
11.03 & 0.01 & 0.011 & 0.005 & 0.01 \\
\hline
\end{tabular}




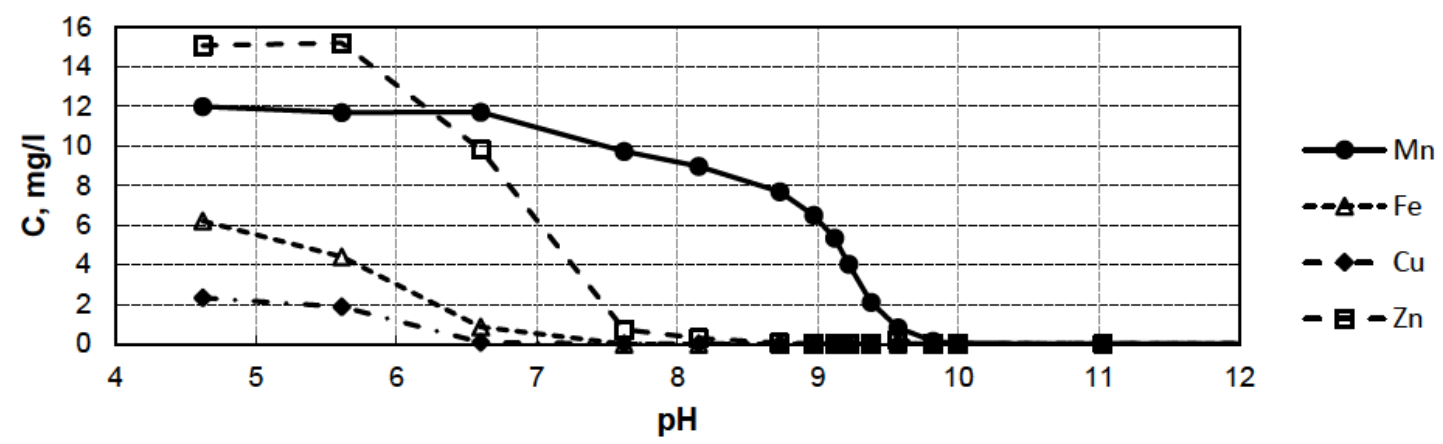

Figure 1: Concentrations of metal ions $\left(\mathrm{Mn}^{2+}, \mathrm{Fe}^{3+}, \mathrm{Cu}^{2+}\right.$ and $\left.\mathrm{Zn}^{2+}\right)$ in mine water samples as a function of $\mathrm{pH}$ (based on the data from Table 1).

\subsection{TD-NMR}

NMR is based on the absorption of energy by nuclei placed in a constant magnet field, which occurs under the resonance of the frequency of nuclei precession and the frequency of their excitation. In the NMR experiments, the registration of interactions of the magnetic moments of atomic nuclei with each other and their surroundings takes place. The main parameters measured by NMR are the spin-spin $R_{2}$ and spin-lattice $R_{1}$ relaxation rates, diffusion coefficients and signal magnitudes in the time domain and the Fourier spectra in the frequency domain. The measured parameters characterize the molecular properties and structure of the materials.

TD-NMR is sensitive to the presence of paramagnetic ions [22], which makes it a potentially applicable technology for measuring metal concentrations in water-intensive processes. In this study, the ${ }^{1} \mathrm{H}$ resonance frequency of the system was $26 \mathrm{MHz}$, and the temperature of the magnet was $30^{\circ} \mathrm{C}$.

Transverse magnetization decays were measured by applying the Carr-Purcell-Meiboom-Gill (CPMG) pulse sequence $[23,24]$. The echo time was $6 \mathrm{~ms}$, and the number of $180^{\circ}$ pulses in the sequence was 600 . The relaxation delay was $6 \mathrm{~s}$, and the number of scans was 4 . The durations of the $90^{\circ}$ and $180^{\circ} \mathrm{RF}$ pulses were 6.5 and $15 \mu \mathrm{s}$, respectively. The transverse magnetization decays of solutions with different $\mathrm{Cu}^{2+}$ concentrations are shown in Figure 2.

The relaxivity of metal ions reflects how the relaxation rates of a solution change as a function of its concentration C. As described in Bloembergen-Purcell-Pound (BPP) theory [22], the effect of paramagnetic ions is strong because their magnetic moments are higher than those of protons $[10,11,22]$. Since a metal ion may affect the two relaxation rates $\left(R_{1}\right.$ and $\left.R_{2}\right)$ individually, there are two corresponding relaxivities, denoted by $r_{1}$ and $r_{2}$. By definition:

$$
R_{1}=r_{1} \cdot C+\text { constant } 1 \text {, }
$$

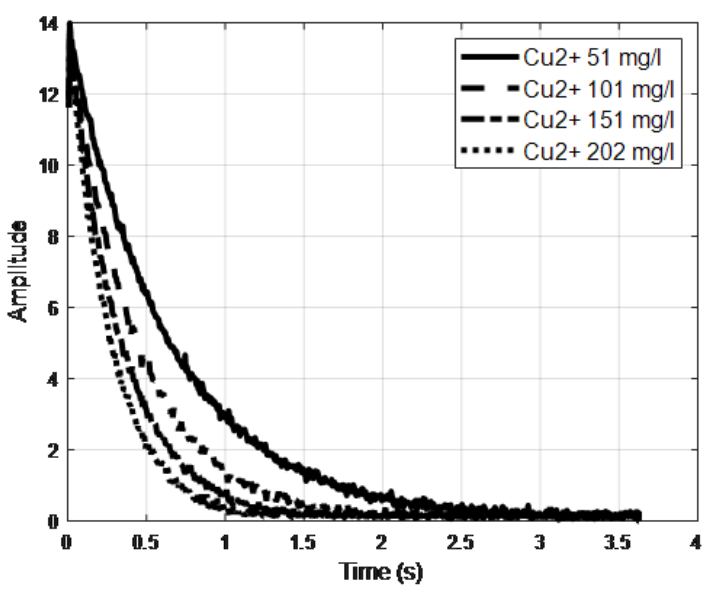

Figure 2: Four transverse magnetization decays of samples with $\mathrm{Cu}^{2+}$.

$$
R_{2}=r_{2} \cdot C+\text { constant } 2 \text {. }
$$

Since $R_{1}$ and $R_{2}$ are given in $\mathrm{s}^{-1}$ and $\mathrm{C}$ is measured in $\mathrm{mg} / \mathrm{l}, r_{1}$ and $r_{2}$ have units of $\mathrm{l} / \mathrm{mg}$ s. constant 1 and constant 2 are the relaxation rates of clean water. The relaxivity depends on the temperature, field strength, and the substance in which the metal ion is dissolved.

Online measurements of metal ions were carried out using a TD-NMR system [25], which has been modified for flowing samples $[15,17,18]$. A MATLAB software script written by the authors was used for controlling the pump, conducting the TD-NMR measurements, fitting the magnetization decays and calculating the relaxation rates.

The measurement procedure was as follows (Figure 3):

1. An input or output sample of a water treatment process was delivered to a container, where it was continuously mixed.

2. The $\mathrm{pH}$ of the sample was measured. 
3. The sample was automatically pumped through the magnet system. The pump was stopped. The transverse magnetization decay was measured.

4. The decay was fitted, and the NMR parameter was solved.

5. A model was applied to determine metals present in the sample.

6. The $R_{2}$ and $r_{2}$ values were used for determination of the metal ion concentrations via Equation 2.

7. A new sample was pumped in.

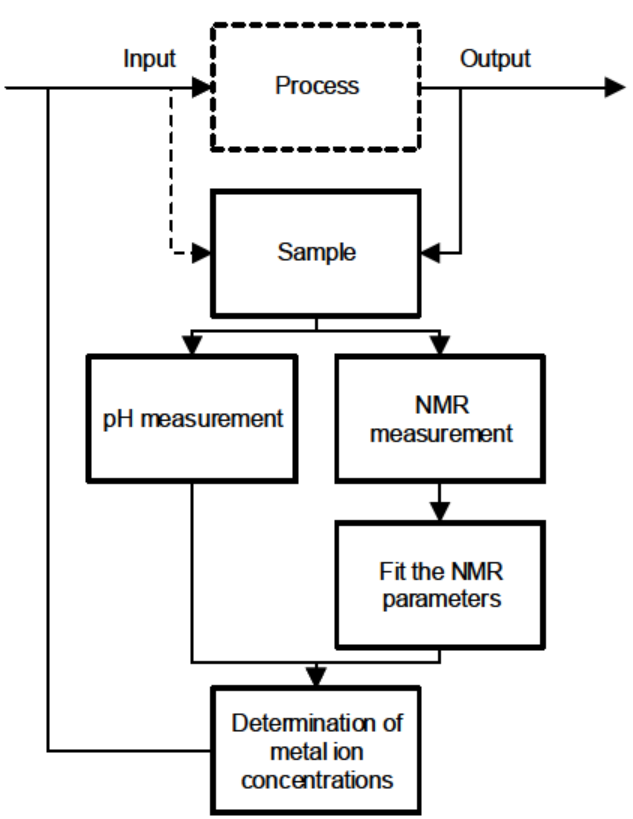

Figure 3: Flow chart of the measurement procedure.

\subsection{Calculation of chemical speciations}

The concentrations of the metal ions as a function of $\mathrm{pH}$ were estimated by means of ChemEQL software [26], which is a computer program for calculating chemical speciation equilibria. The experimental concentrations of the metal ions at pH 4.62 (see Table 1) were used as the initial ion concentrations. Then, the concentration at each $\mathrm{pH}$ was estimated by the ChemEQL software; finally, the total relaxation rate $R_{2}$ results were calculated as a function of $\mathrm{pH}$ using the following equation:

$$
R_{2}=\sum_{i=1}^{n} r_{2 i} C_{i}
$$

where $n$ is the number of ions, the $r_{2 i}$ values are from Table 2 and the concentration $C$ is calculated by the software.

\section{Results}

\subsection{Relaxivities of metals}

In this paper, we focus on the relaxation rate $R_{2}$ and relaxivity $r_{2}$ results for several metal ions. The relaxivity measurements were conducted for the following ions: $\mathrm{Mn}^{2+}$, $\mathrm{Fe}^{3+}, \mathrm{Fe}^{2+}, \mathrm{Cu}^{2+}, \mathrm{Zn}^{2+}$ and $\mathrm{Ni}^{2+}$. In addition, the $\mathrm{Cu}^{2+}$ relaxivities were determined using two different chemicals. As an example, the correlation between the chemical concentration of $\mathrm{Fe}^{3+}$ and its relaxation rate $R_{2}$ is presented in Figure 4, showing a very high correlation. The results of all the ions are collected in Table 2. In summary, the measurements for the concentrations of single metals are very accurate.

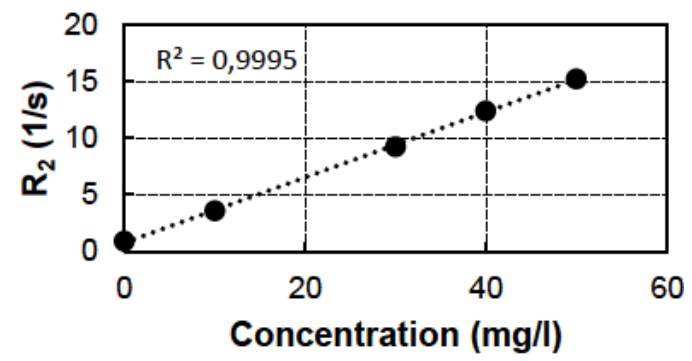

Figure 4: Relaxation rate $R_{2}$ as a function of $\mathrm{Fe}^{3+}$ concentration.

Table 2: Relaxivity $r_{2}$ results for metal ions obtained from a waterbased solution by TD-NMR. Numbers in parentheses show the errors of the relaxivities.

\begin{tabular}{cc}
\hline Metal ion & Relaxivity $\mathbf{r}_{\mathbf{2}}(\mathrm{l} / \mathbf{m g ~ s})$ \\
\hline $\mathrm{Mn}^{2+}$ & $0.81765(226)$ \\
$\mathrm{Fe}^{3+}$ & $0.28799(365)$ \\
$\mathrm{Fe}^{2+}$ & $0.01194(1)$ \\
$\mathrm{Cu}^{2+}\left(\mathrm{CuSO}_{4}\right)$ & $0.01493(10)$ \\
$\mathrm{Cu}^{2+}\left(\mathrm{CuCl}_{2}\right)$ & $0.01443(14)$ \\
$\mathrm{Ni}^{2+}$ & $0.01239(9)$ \\
$\mathrm{Zn}^{2+}$ & $0.00069(1)$ \\
\hline
\end{tabular}

\subsection{Relaxation rates of metal mixtures}

The participation of simple mixtures of metal ions was studied by TD-NMR in the laboratory. The relaxation rates of two mixtures of $\mathrm{Mn}^{2+}$ and $\mathrm{Fe}^{3+}$ ions in different concen- 


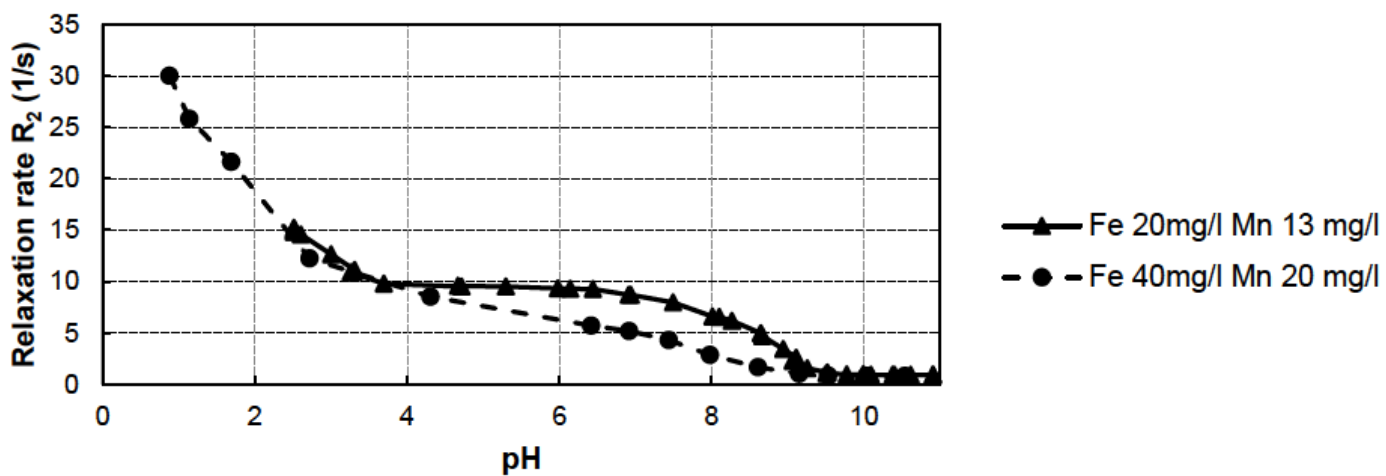

Figure 5: Relaxation rate $R_{2}$ results for two different mixtures of $\mathrm{Mn}^{2+}$ and $\mathrm{Fe}^{3+}$ as a function of $\mathrm{pH}$.

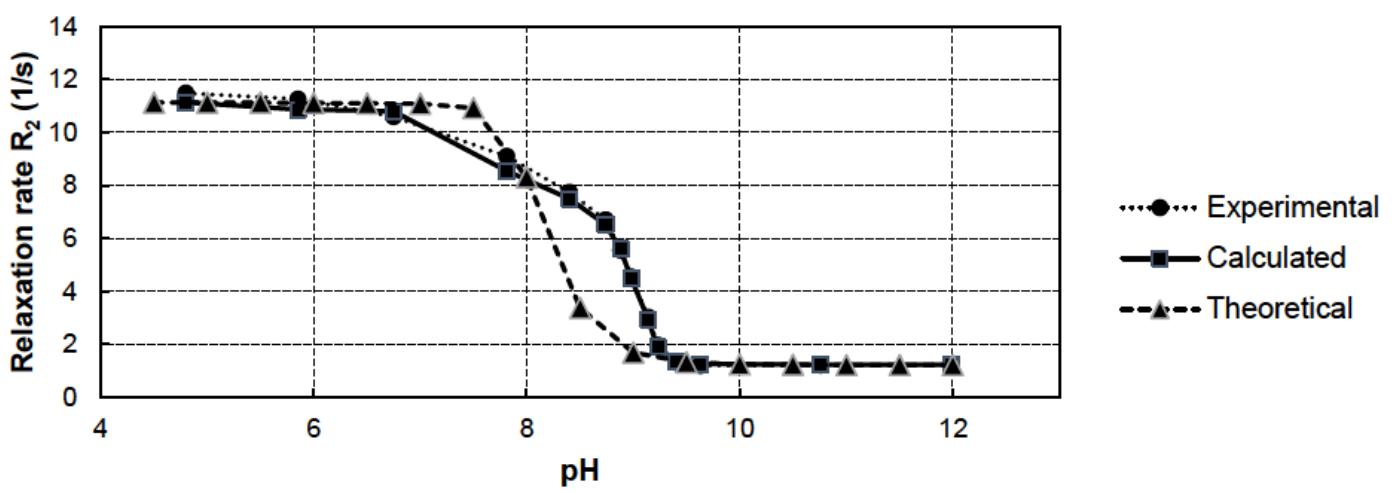

Figure 6: Total relaxation rate $R_{2}$ results for mixtures of $\mathrm{Mn}^{2+}, \mathrm{Cu}^{2+}, \mathrm{Fe}^{3+}, \mathrm{Fe}^{2+}$ and $\mathrm{Zn}^{2+}$ in mining water as a function of $\mathrm{pH}$.

trations $\left(\mathrm{Fe}^{3+} 20 \mathrm{mg} / \mathrm{l}+\mathrm{Mn}^{2+} 13 \mathrm{mg} / \mathrm{l}\right.$ and $\mathrm{Fe}^{3+} 40 \mathrm{mg} / \mathrm{l}+$ $\left.\mathrm{Mn}^{2+} 20 \mathrm{mg} / \mathrm{l}\right)$ as a function of $\mathrm{pH}$ are shown in Figure 5.

Remarkably, the relaxation rates of these two mixtures behave quite differently at $\mathrm{pH}$ values of 4-10. This is probably due to a change in the redox value of the mixtures. In the case of the latter mixture $\left(\mathrm{Fe}^{3+} 40 \mathrm{mg} / \mathrm{l}+\mathrm{Mn}^{2+} 20\right.$ $\mathrm{mg} / \mathrm{l}$ ), the redox potential eventually increases so much that the precipitation of $\mathrm{Mn}^{2+}$ begins at a lower $\mathrm{pH}$. The main conclusion to be drawn here is that the behaviour of mixtures of metal ions is, in general, observable by the TD-NMR method, even if the behaviour is controlled by changes in the redox potential of the solution.

\subsection{Mining water measurements}

In real-world conditions, metals in water are typically bound within mixtures of several metal components. Therefore, the relaxation rates of a real mining water sample were also measured, and the results were plotted as a function of $\mathrm{pH}$. The experimental relaxation rates of the water are shown in Figure 6. ("Experimental"). The Mn, Fe, $\mathrm{Cu}$ and $\mathrm{Zn}$ concentrations of the samples were also measured by the XRF method. Using the concentration values in Table 1 and the relaxivities presented in Table 2, the corresponding relaxation rates were calculated using Equation 3 (Figure 6; "Calculated"). The error between the experimental and calculated $R_{2}$ values was $2.9 \%$, which indicates the good accuracy of the NMR method. It also confirms that the NMR effects of $\mathrm{Al}$ and $\mathrm{Mg}$ ions are small compared to those of other paramagnetic ions.

In addition, the concentrations of metal ions were estimated by means of ChemEQL software [26]. Again, using these $r_{2}$ values and Equation 3, the theoretical relaxation rates were calculated (Figure 6; “Theoretical”). All three determinations are in relativity good agreement. The results show that among the measured, calculated, and theoretical results, there is a more dramatic decrease in the relaxation rate at lower $\mathrm{pH}$ values for the NMR measurements, whereas the decrease in the relaxation rate of the theoretical calculations starts later. The results indicate that the difference between the experimental and theoretical values may arise from the redox reactions of the experimental samples. 

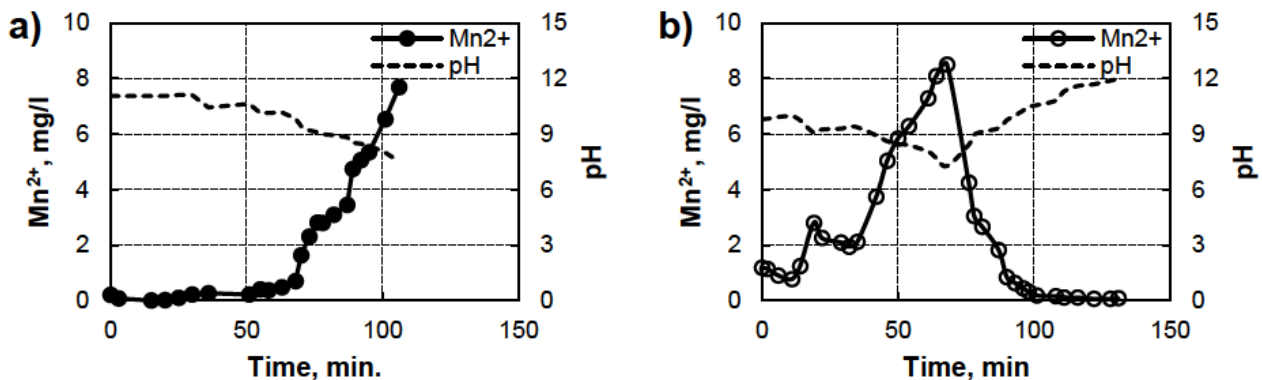

Figure 7: Concentration of $\mathrm{Mn}^{2+}$ in the output purified mining water as a function of $\mathrm{pH}$, measured by TD-NMR, on day 1 (a) and day 2 (b).

\subsection{Online measurements}

The approach was tested in a real process: the estimation of metal ion contents in mining water during continuous purification. In the test shown in Figure 7, the change in $\mathrm{pH}$ of the output of this process ranges between 7 and 10. According to the data from Table 1, the concentration of only $\mathrm{Mn}^{2+}$ can be detected. All the other metals are minor concentrations at these $\mathrm{pH}$ levels, so they could be neglected. $\mathrm{The}^{2+}$ concentration was calculated using Equation 2, and the relaxivity of $\mathrm{Mn}^{2+}$ is presented in Table $2 . R_{2}=1.27$ $\mathrm{s}^{-1}$ at $\mathrm{pH}=11.05$ was used as constant 2 in Equation 2.

\section{Discussion}

In this paper, TD-NMR technology was applied for monitoring the concentrations of $\mathrm{Mn}^{2+}, \mathrm{Cu}^{2+}, \mathrm{Fe}^{3+}, \mathrm{Fe}^{2+}, \mathrm{Zn}^{2+}$ and $\mathrm{Ni}^{2+}$ ions in water-based solutions. These paramagnetic ions are often the principal metal components in mining waters. XRF measurements and chemical speciation equilibria calculations were compared to the TD-NMR results. The results show the potential of TD-NMR for monitoring metal ion concentrations in process and mining water treatment.

Determination of the concentration of single paramagnetic ions in aqueous solution by TD-NMR relaxation is a robust approach, as previously demonstrated [9-11]. Moreover, TD-NMR was applied to the online monitoring of the precipitation of metals in real mines [14], and several investigations were performed in situ by measurements of samples placed in standard NMR tubes or by single-sided NMR [9, 12]. However, TD-NMR technology has rarely been applied to the online determination of chemical concentrations in industrial environments.

The measurement of metals in liquid is a new field of research and business because reliable online determination methods for different kinds of metal ions in process and mining waters do not exist. The results of this work and earlier papers $[14,16-18]$ indicate that the online TDNMR method is not sensitive to fouling. Specifically, TDNMR was successfully applied for the determination of the content of solids in black liquor at a pulp mill in a longterm test [18]. This property may provide a cost benefit due to lower maintenance costs. When considering the potential for producing commercial products, the results of this approach may offer a starting point for achieving cost savings by reducing both energy consumption and chemical consumption.

Online measurement of metal concentrations will be the first step towards more efficient and optimized control of water-intensive processes. Better measurement and control enable the optimization of processes, making it possible to achieve optimal purification, energy savings via the optimization of mixing processes, and chemical savings via the optimization of chemical dosage.

In the long term, better management of metals in liquid makes it possible to reduce the environmental impacts of water-intensive processes; thus, the well-being of the people, plants and other point sources of release in a mine's sphere of influence may improve.

\section{Conclusion}

The measuring system presented in this paper is applicable for continuous real-time monitoring of the concentrations of a number of paramagnetic ions in water and controlling the water purification processes. TD-NMR is a nonfouling technique and might be beneficial for use in harsh industrial environments, such as mines, and municipal or industrial wastewater treatment processes. The performance of the system in various water-intensive processes will be further tested.

Acknowledgement: This research is a part of the QualityMeas projects, which are funded by the Centre for Eco- 
nomic Development, Transport and the Environment and four companies.

\section{References}

[1] Fu F, Wang Q. Removal of heavy metal ions from wastewaters: a review. J Environ Manage. 2011 Mar;92(3):407-18.

[2] Nasiri EF, Kebria DY, Qaderi F. An Experimental Study on the Simultaneous Phenol and Chromium Removal From Water Using Titanium Dioxide Photocatalyst, Civil. Eng J (NY). 2018 Mar;4(3):58593.

[3] Beidokhti MZ, Naeeni ST. AbdiGhahroudi M.S., Biosorption of Nickel (II) from Aqueous Solutions onto Pistachio Hull Waste as a Low-Cost Biosorbent, Civil. Eng J (NY). 2019 Feb;5(2):447-57.

[4] Nkansah MA, Shamsu-Deen M, Opoku F. Phytocompounds, Heavy Metal and Mineral Contents in honey Samples from Selected Markets in the Kumasi Metropolis. Emerging Science Journal. 2018 Oct;2(5):287-94.

[5] Gomes B.F., da Silva Burato J.S., Silva Lobo C.M, and Colnago L.A., Use of the Relaxometry Technique for Quantification of Paramagnetic lons in Aqueous Solutions and a Comparison with Other Analytical Methods, Hindawi Publishing Corporation International Journal of Analytical Chemistry Volume 2016, Article ID 8256437 , 5 pages, DOI: https://doi.org/10.1155/2016/8256437.

[6] Dalitz F, Cudaj M, Maiwald M, Guthausen G. Process and reaction monitoring by low-field NMR spectroscopy. Prog Nucl Magn Reson Spectrosc. 2012 Jan;60:52-70.

[7] Mitchell J, Gladden LF, Chandrasekera TC, Fordham EJ. Low-field permanent magnets for industrial process and quality control. Prog Nucl Magn Reson Spectrosc. 2014 Jan;76:1-60.

[8] Blümich B, Casanova F, Appelt S. NMR at low magnetic fields. Chem Phys Lett. 2009;477(4-6):231-40.

[9] Mitreiter I, Oswald SE, Stallmach F. Investigation of Iron(III)Release in the Pore Water of Natural Sands by NMR Relaxometry. Open Magn Reson J. 2010;3(1):46-51.

[10] Kock FV, Machado MP, Athayde GP, Colnago LA, Barbosa LL. Quantification of paramagnetic ions in solution using time domain NMR. PROS and CONS to optical emission spectrometry method. Microchem J. 2018;137:204-7.

[11] Cobra PF, Gomes BF, Mitre CI, Barbosa LL, Marconcini LV, Colnago LA. Measuring the solubility product constant of paramagnetic cations using time-domain nuclear magnetic resonance relaxometry. Microchem J. 2015;121:14-7.
[12] Gomes BF, Nunes LM, Lobo CM, Carvalho AS, Cabeça LF, Colnago LA. In situ analysis of copper electrodeposition reaction using unilateral NMR sensor. J Magn Reson. 2015 Dec;261:83-6.

[13] Colnago LA, Andrade FD, Souza AA, Azeredo RB, Lima AA, Cerioni LM, et al. Why is Inline NMR Rarely Used as Industrial Sensor? Challenges and Opportunities. Chem Eng Technol. 2014;37(2):191-203.

[14] Nikolskaya E, Liukkonen M, Kankkunen J, Hiltunen Y. A nonfouling online method for monitoring precipitation of metal ions in mine waters, IFAC Proceedings Volumes, 2015, 48-17, 98-101, DOI:https://doi.org/10.1016/j.ifacol.2015.10.085.

[15] Nikolskaya E, Hiltunen Y. Determination of Carbon Chain Lengths of Fatty Acid Mixtures by Time Domain NMR. Appl Magn Reson. 2018;49(2):185-93.

[16] Nikolskaya E, Hiltunen Y. Molecular Properties of Fatty Acid Mixtures Estimated by Online Time-Domain NMR. Appl Magn Reson. 2019;50(1-3):159-70.

[17] Raunio J, Nikolskaya E, Hiltunen Y. On-line monitoring of cationic starch gelatinization and retrogradation by $1 \mathrm{H}$ NMR-relaxometry. Nord Pulp Paper Res J. 2018;33(4):625-31.

[18] Nikolskaya E, Janhunen P, Haapalainen M, Hiltunen Y. Solids content of black liquor measured by online Time-Domain NMR. Appl Sci (Basel). 2019;9(10):2169.

[19] Maiwald M, Gräßer P, Wander L, Zientek N, Guhl S, Meyer K, et al. Strangers in the Night-Smart Process Sensors in Our Current Automation Landscape. Proceedings. 2017;1(4):628.

[20] Eisen K, Eifert T, Herwig C, Maiwald M. Current and future requirements to industrial analytical infrastructure-part 1: process analytical laboratories. Anal Bioanal Chem. 2020 Apr;412(9):202735.

[21] Eifert T, Eisen K, Maiwald M, Herwig C. Current and future requirements to industrial analytical infrastructure-part 2: smart sensors. Anal Bioanal Chem. 2020 Apr;412(9):2037-45.

[22] Bloembergen N, Purcell E, Pound R. Relaxation Effects in Nuclear Magnetic Resonance Absorption. Phys Rev. 1948;73(7):679-712.

[23] Carr H, Purcell E. Effects of diffusion on free precession in nuclear magnetic resonance experiments. Phys Rev. 1954;94(3):630-8.

[24] Meiboom S, Gill D. Modified Spin-Echo Method for Measuring Nuclear Relaxation Times. Rev Sci Instrum. 1958;29(8):688-91.

[25] Web pages of Resonance Systems, http://www.nmr-design.com, accessed 2018, March 28.

[26] Web pages of Eawag - Swiss Federal Institute of Aquatic Science and Technology, https://www.eawag.ch/en/departme nt/surf/projects/chemeql/, accessed 2019, March 28. 